\title{
Inverse Nodal Problems for Impulsive Sturm-Liouville Equation with Boundary Conditions Depending on the Parameter
}

\author{
Baki Keskin and A. Sinan Ozkan* \\ Department of Mathematics, Faculty of Science, Cumhuriyet University, 58140, Sivas, Turkey \\ Email: sozkan@cumhuriyet.edu.tr
}

\begin{abstract}
In this work, the Sturm-Liouville problem with boundary conditions depending rationally on the spectral parameter is studied. We give a uniqueness theorem and algorithm to reconstruct the potential of the problem from nodal points (zeros of eigenfunctions).
\end{abstract}

Keywords: Sturm-Liouville equation, inverse nodal problem, parameter dependent boundary condition, discontinuity condition.

\section{Introduction}

We consider the boundary value problem $L$ generated by the regular Sturm-Liouville equation

$$
\ell y:=-y^{\prime \prime}+q(x) y=\lambda y, \quad x \in(0,1)
$$

subject to the boundary conditions

$$
\begin{aligned}
& U(y):=a(\lambda) y^{\prime}(0)-b(\lambda) y(0)=0 \\
& V(y):=c(\lambda) y^{\prime}(1)-d(\lambda) y(1)=0
\end{aligned}
$$

and the jump conditions

$$
\left\{\begin{array}{c}
y\left(\frac{1}{2}+0\right)=\alpha y\left(\frac{1}{2}-0\right) \\
y^{\prime}\left(\frac{1}{2}+0\right)=\alpha^{-1} y^{\prime}\left(\frac{1}{2}-0\right),
\end{array}\right.
$$

where $\lambda$ is the spectral parameter; $q(x)$ is a real-valued function from the class $L_{2}(0,1) ; \alpha$ is a positive real constant; $a(\lambda), b(\lambda), c(\lambda)$ and $d(\lambda)$ are real polynomials such that

$$
\begin{aligned}
& a(\lambda)=\sum_{j=0}^{m} a_{j} \lambda^{j}, b(\lambda)=\sum_{j=0}^{m} b_{j} \lambda^{j}, \\
& c(\lambda)=\sum_{j=0}^{r} c_{j} \lambda^{j}, d(\lambda)=\sum_{j=0}^{r} d_{j} \lambda^{j},
\end{aligned}
$$

Without loss of generality, we assume that $a_{m}=c_{r}=1$ and $\int_{0}^{1} q(x) \mathrm{d} x=0$, and define $f=\frac{a(\lambda)}{b(\lambda)}$.

The values of the parameter $\lambda$ for which $L$ has nonzero solutions, are called eigenvalues and the corresponding nontrivial solutions are called eigenfunctions.

Spectral problems for various differential equation with the eigen-dependent-boundary conditions have been well studied. Inverse problems for the special case when $f$ is an affine function on $\lambda$ were solved in [11]. The case when $f$ is a more general rational function of $\lambda$ is difficult. In [1]-[4], [8], [16], [13], [19] and [23], various spectral problems with rational conditions were studied.

Inverse spectral problems for Sturm-Liouville operator with the discontinuity conditions, like (4), were studied in [7], [12] and references therein.

The inverse nodal problem, which is different from the classical inverse spectral theory of Gelfand and Levitan [10], was initiated by McLaughlin [15]. Later, Hald and McLaughlin [13] and Browne and 
Sleeman [5] proved that it is sufficient to know the nodal points to uniquely determine the potential function of the regular Sturm-Liouville problem. Yang gave an algorithm to recover $q$ from dense subset of nodal points[20]. Recently, the inverse nodal Sturm-Liouville problems has been investigated by several authors [5], [6], [13], [15], [17], [18], [21] and [22].

In the present paper, we investigate an impulsive Sturm-Liouville operator and give a uniqueness theorem to reconstruct the potential of the problem from nodal points.

\section{Preliminaries}

Let $\varphi(x, \lambda)$ be the solution of $(1)$, satisfying the initial conditions

$$
\varphi(0, \lambda)=a(\lambda), \quad \varphi^{\prime}(0, \lambda)=b(\lambda)
$$

and the jump conditions (4). Moreover, the following integral equations of the solution hold for $x<\frac{1}{2}$

$$
\begin{aligned}
\varphi(x, \lambda)= & a(\lambda) \cos \sqrt{\lambda} x+b(\lambda) \frac{\sin \sqrt{\lambda} x}{\sqrt{\lambda}} \\
& +\int_{0}^{x} \frac{\sin \sqrt{\lambda}(x-t)}{\sqrt{\lambda}} q(t) \varphi(t, \lambda) \mathrm{d} t,
\end{aligned}
$$

for $x>\frac{1}{2}$

$$
\begin{aligned}
\varphi(x, \lambda)= & \alpha^{+}\left[a(\lambda) \cos \sqrt{\lambda} x+b(\lambda) \frac{\sin \sqrt{\lambda} x}{\sqrt{\lambda}}\right] \\
& +\alpha^{-}\left[a(\lambda) \cos \sqrt{\lambda}(1-x)+b(\lambda) \frac{\sin \sqrt{\lambda}(1-x)}{\sqrt{\lambda}}\right] \\
& +\int_{0}^{1 / 2}\left[\alpha^{+} \frac{\sin \sqrt{\lambda}(x-t)}{\sqrt{\lambda}}+\alpha^{-} \frac{\sin \sqrt{\lambda}(1-x-t)}{\sqrt{\lambda}}\right] q(t) \varphi(t, \lambda) \mathrm{d} t \\
& +\int_{1 / 2}^{x} \frac{\sin \sqrt{\lambda}(x-t)}{\sqrt{\lambda}} q(t) \varphi(t, \lambda) \mathrm{d} t
\end{aligned}
$$

where $\alpha^{ \pm}=\frac{1}{2}\left(\alpha \pm \frac{1}{\alpha}\right)$. Using these equations, we prove that the following asymptotic relations are valid for $|\lambda| \rightarrow \infty$,

for $x<\frac{1}{2}$

$$
\varphi(x, \lambda)=\lambda^{m}\left\{\cos \sqrt{\lambda} x+\frac{\sin \sqrt{\lambda} x}{\sqrt{\lambda}}\left(b_{m}+\frac{1}{2} \int_{0}^{x} q(t) \mathrm{d} t\right)+o\left(\frac{1}{\sqrt{\lambda}} \exp \tau x\right)\right\}
$$

for $x>\frac{1}{2}$

$$
\begin{aligned}
\varphi(x, \lambda)= & \lambda^{m}\left\{\alpha^{+} \cos \sqrt{\lambda} x+\alpha^{-} \cos \sqrt{\lambda}(1-x)\right\}+ \\
& +\lambda^{m-\frac{1}{2}}\left\{\alpha^{+} I_{1}(x) \sin \sqrt{\lambda} x+\alpha^{-} I_{2}(x) \sin \sqrt{\lambda}(1-x)\right\} \\
& +o\left(\lambda^{m-\frac{1}{2}} \exp \tau x\right)
\end{aligned}
$$

where

$$
\begin{aligned}
& I_{1}(x)=b_{m}+\frac{1}{2} \int_{0}^{x} q(t) \mathrm{d} t \\
& I_{2}(x)=b_{m}+\frac{1}{2} \int_{0}^{1 / 2} q(t) \mathrm{d} t-\frac{1}{2} \int_{1 / 2}^{x} q(t) \mathrm{d} t .
\end{aligned}
$$


and $\tau=|\operatorname{Im} \sqrt{\lambda}|$.

Consider the function

$$
\Delta(\lambda):=c(\lambda) \varphi^{\prime}(1, \lambda)-d(\lambda) \varphi(1, \lambda) .
$$

$\Delta(\lambda)$ is called characteristic function of the problem $L$. It is obvious that $\Delta(\lambda)$ is an entire function and its zeros, namely $\left\{\lambda_{n}\right\}_{n \geq 0}$, are eigenvalues of the problem $L$. Moreover, the following relation holds.

$$
\Delta(\lambda)=-\alpha^{+} \lambda^{m+r}\left\{\sqrt{\lambda} \sin \sqrt{\lambda}-w_{1} \cos \sqrt{\lambda}+w_{2}+o(\exp \tau)\right\} .
$$

It can be shown using classical methods in the similar studies that the sequence $\left\{\lambda_{n}\right\}_{n \geq 0}$ satisfies the following asymptotic relation for $n \rightarrow \infty$ :

$$
\sqrt{\lambda_{n}}=(n-m-r) \pi+\frac{\left(w_{1}-(-1)^{n-m-r} w_{2}\right)}{(n-m-r) \pi}+o\left(\frac{1}{n}\right)
$$

where $w_{1}=I_{1}(1)-d r$ and $w_{2}=\frac{\alpha^{-}}{\alpha^{+}}\left(I_{2}(1)+d_{r}\right)$.

Let $\varphi\left(x, \lambda_{n}\right)$ be the eigenfunction corresponding to the eigenvalue $\lambda_{n}$.

Lemma 2.1. $\varphi\left(x, \lambda_{n}\right)$ has exactly $n-m-r$ nodes $\left\{x_{n}^{j}: j=\overline{0, n-m-r-1}\right\}$ in $(0,1)$ for sufficiently large $n$. The numbers $\left\{x_{n}^{j}\right\}$ satisfy the following asymptotic formulae for $x_{n}^{j} \in\left(0, \frac{1}{2}\right)$

$$
x_{n}^{j}=\left\{\begin{array}{c}
\frac{(j+1 / 2)}{n-m-r}+\frac{I_{1}\left(x_{n}^{j}\right)}{(n-m-r)^{2} \pi^{2}}-\frac{\left(w_{1}-w_{2}\right)}{(n-m-r)^{2} \pi^{2}} \frac{(j+1 / 2)}{n-m-r} \\
+o\left(\frac{1}{n^{2}}\right), \text { for } n-m-r=2 k \\
\frac{(j+1 / 2)}{n-m-r}+\frac{I_{1}\left(x_{n}^{j}\right)}{(n-m-r)^{2} \pi^{2}}-\frac{\left(w_{1}+w_{2}\right)}{(n-m-r)^{2} \pi^{2}} \frac{(j+1 / 2)}{n-m-r} \\
+o\left(\frac{1}{n^{2}}\right), \text { for } n-m-r=2 k+1
\end{array}\right.
$$

and for $x_{n}^{j} \in\left(\frac{1}{2}, 1\right)$

$$
x_{n}^{j}=\left\{\begin{array}{c}
\frac{(j+1 / 2)}{n-m-r}+\frac{w_{1}-w_{2}}{(n-m-r)^{2} \pi^{2}} \frac{(j+1 / 2)}{n-m-r}+\frac{1}{2(n-m-r)^{2} \pi^{2}} \int_{0}^{x} q(t) \mathrm{d} t \\
\quad+\frac{\rho_{0}}{(n-m-r)^{2} \pi^{2}}+o\left(\frac{1}{n^{2}}\right), \text { for } n-m-r=2 k \\
\frac{(j+1 / 2)}{n-m-r}+\frac{w_{1}+w_{2}}{(n-m-r)^{2} \pi^{2}} \frac{(j+1 / 2)}{n-m-r}+\frac{1}{2(n-m-r)^{2} \pi^{2}} \int_{0}^{x} q(t) \mathrm{d} t \\
\quad+\frac{\rho_{1}}{(n-m-r)^{2} \pi^{2}}+o\left(\frac{1}{n^{2}}\right), \text { for } n-m-r=2 k+1
\end{array}\right.
$$

where

$$
\begin{aligned}
& \rho_{0}=\frac{\alpha^{-}}{2 \alpha^{+}}\left(\int_{1 / 2}^{1} q(t) \mathrm{d} t-\int_{0}^{1 / 2} q(t) \mathrm{d} t-2 d_{r}\right)+\frac{\alpha^{+}-\alpha^{-}}{\alpha^{+}} b_{m}, \\
& \rho_{1}=\frac{\alpha^{-}}{2 \alpha^{+}}\left(\frac{\alpha^{+}+\alpha^{-}}{\alpha^{+}-\alpha^{-}}\right)\left(\int_{0}^{1 / 2} q(t) \mathrm{d} t-\int_{1 / 2}^{1} q(t) \mathrm{d} t+2 d_{r}\right)+\frac{\left(\alpha^{+}\right)^{2}-\left(\alpha^{-}\right)^{2}}{\left(\alpha^{+}-\alpha^{-}\right) \alpha^{+}} b_{m} .
\end{aligned}
$$

Proof. It can be seen from (8), (9) and oscilation theorem that the function $\varphi\left(x, \lambda_{n}\right)$ has exactly $n-m-r$ zeros in the interval $(0,1)$ for sufficiently large $n$. Using $(8)$ and $(9)$ again, we get the following asymptotic formulae

$$
\begin{gathered}
\varphi\left(x, \lambda_{n}\right)=\lambda_{n}^{m}\left\{\cos \sqrt{\lambda_{n}} x+\frac{\sin \sqrt{\lambda_{n}} x}{\sqrt{\lambda_{n}}} I_{1}(x)+o\left(\frac{\exp \tau_{n} x}{\sqrt{\lambda_{n}}}\right)\right\} \text { for } x<\frac{1}{2}, \\
\varphi\left(x, \lambda_{n}\right)=\lambda_{n}^{m}\left\{\alpha^{+} \cos \sqrt{\lambda_{n}} x+\alpha^{-} \cos \sqrt{\lambda_{n}}(1-x)\right\} \\
+\lambda^{m-\frac{1}{2}}\left\{\alpha^{+} I_{1}(x) \sin \sqrt{\lambda_{n}} x+\alpha^{-} I_{2}(x) \sin \sqrt{\lambda_{n}}(1-x)\right\} \\
+o\left(\lambda^{m-\frac{1}{2}} \exp \tau_{n} x\right) \text { for } x>\frac{1}{2} .
\end{gathered}
$$


From

$$
\begin{aligned}
& 0=\varphi\left(x_{n}^{j}, \lambda_{n}\right) \\
& =\lambda_{n}^{m}\left\{\alpha^{+} \cos \sqrt{\lambda_{n}} x_{n}^{j}+\alpha^{-} \cos \sqrt{\lambda_{n}}\left(1-x_{n}^{j}\right)\right\} \\
& +\lambda^{m-\frac{1}{2}}\left\{\alpha^{+} I_{1}\left(x_{n}^{j}\right) \sin \sqrt{\lambda_{n}} x_{n}^{j}+\alpha^{-} I_{2}\left(x_{n}^{j}\right) \sin \sqrt{\lambda_{n}}\left(1-x_{n}^{j}\right)\right\}+o\left(\lambda^{m-\frac{1}{2}} \exp \tau_{n} x_{n}^{j}\right),
\end{aligned}
$$

we get

$$
\begin{aligned}
\tan & \left(\sqrt{\lambda_{n}} x-\frac{\pi}{2}\right) \\
& =\frac{(-1)^{n-m-r}\left(w_{1}-(-1)^{n-m-r} w_{2}\right) \alpha^{-}+\alpha^{+} I_{1}(x)-(-1)^{n-m-r} \alpha^{-} I_{2}(x)}{\left(\alpha^{+}+(-1)^{n-m-r} \alpha^{-}\right)(n-m-r) \pi}+o\left(\frac{1}{n}\right),
\end{aligned}
$$

for $x_{n}^{j}>\frac{1}{2}$. Taylor's formula for the arctangent yields

$$
\begin{aligned}
x_{n}^{j}= & \frac{(j+1 / 2)}{n-m-r}+\frac{w_{1}-(-1)^{n-m-r} w_{2}}{(n-m-r)^{2} \pi^{2}} \frac{(j+1 / 2)}{n-m-r} \\
& +\frac{(-1)^{n-m-r}\left(w_{1}-(-1)^{n-m-r} w_{2}\right) \alpha^{-}+\alpha^{+} I_{1}\left(x_{n}^{j}\right)-(-1)^{n-m-r} \alpha^{-} I_{2}\left(x_{n}^{j}\right)}{\left(\alpha^{+}+(-1)^{n-m-r} \alpha^{-}\right)(n-m-r)^{2} \pi^{2}}+o\left(\frac{1}{n^{2}}\right) .
\end{aligned}
$$

The last equality is the proof of (14). The equation (13) can be proved similarly.

Let $X=X_{0} \cup X_{1}$ be the set of nodal points such that $X_{0}=\left\{x_{n}^{j}: n-m-r=2 s, s \in \mathbb{Z}\right\}, X_{1}=$ $\left\{x_{n}^{j}: n-m-r=2 s+1, s \in \mathbb{Z}\right\}$. For each fixed $x \in[0,1]$ and $k \in\{0,1\}$, there exists a sequence $\left(x_{n}^{j(n)}\right) \subset X_{k}$ which converges to $x$. Therefore, from Lemma 2.1, we can show the following limits are exist and finite

$$
\lim _{n \rightarrow \infty}(n-m-r)^{2} \pi^{2}\left(x_{n}^{j(n)}-\frac{\left(j(n)+\frac{1}{2}\right) \pi}{n-m-r}\right)=f_{k}(x),
$$

where

$$
f_{k}(x)=\left\{\begin{array}{l}
\frac{1}{2} \int_{0}^{x} q(t) \mathrm{d} t-\left(w_{1}-(-1)^{k} w_{2}\right) x+b_{m} \text { for } x<\frac{1}{2} \\
\frac{1}{2} \int_{0}^{x} q(t) \mathrm{d} t-\left(w_{1}-(-1)^{k} w_{2}\right) x+\rho_{k} \text { for } x>\frac{1}{2}
\end{array}\right.
$$

Theorem 2.2. The given nodal sets $X_{0}$ or $X_{1}$ uniquely determine the potential $q(x)$, a.e. on $(0,1)$ and the coefficients $b_{m}$ and $d_{r}$ of the boundary conditions. The potential $q(x)$ and the constants $b_{m}$ and $d_{r}$ can be constructed by the following formulae:

1- For each fixed $x \in[0,1]$, choose a sequence $\left(x_{n}^{j(n)}\right) \subset X$ such that $\lim _{n \rightarrow \infty} x_{n}^{j(n)}=x$;

2- Find the function $f_{k}(x)$ from the equation (16) and calculate

$$
\begin{aligned}
q(x) & =2\left[f_{k}^{\prime}(x)-f_{k}(1)+f_{k}(0)+f_{k}\left(\frac{1}{2}+0\right)-f_{k}\left(\frac{1}{2}-0\right)\right] \\
b_{m}=f_{k}(0) & \\
d_{r}=f_{k}(1)-f_{k}\left(\frac{1}{2}+0\right)+f_{k}\left(\frac{1}{2}-0\right) & \\
& -(-1)^{k} \frac{\alpha^{-}}{\alpha^{+}}\left(b_{m}+\int_{0}^{1 / 2} q(t) \mathrm{d} t\right)
\end{aligned}
$$


Proof. Direct calculations in (13), (14) and (16) yield

$$
\begin{aligned}
b_{m} & =f_{k}(0), \\
q(x) & =2\left[f_{k}^{\prime}(x)-\left(w_{1}-(-1)^{k} w_{2}\right)\right] \\
w_{1}-(-1)^{k} w_{2} & =f_{k}(1)-c_{k} \\
c_{k} & =f_{k}\left(\frac{1}{2}+0\right)-f_{k}\left(\frac{1}{2}-0\right)+b_{m} \\
d_{r} & =f_{k}(1)-f_{k}\left(\frac{1}{2}+0\right)+f_{k}\left(\frac{1}{2}-0\right)+ \\
-(-1)^{k} \frac{\alpha^{-}}{\alpha^{+}} & \left(b_{m}+\int_{0}^{1 / 2} q(t) \mathrm{d} t\right)
\end{aligned}
$$

This completes the proof.

Example 2.3. Consider the BVP

$$
L:\left\{\begin{array}{c}
\ell y:=-y^{\prime \prime}+q(x) y=\lambda y, \quad x \in(0,1), \\
a(\lambda) y^{\prime}(0)-b(\lambda) y(0)=0 \\
c(\lambda) y^{\prime}(1)-d(\lambda) y(1)=0 \\
y\left(\frac{1}{2}+0\right)=\alpha y\left(\frac{1}{2}-0\right) \\
y^{\prime}\left(\frac{1}{2}+0\right)=\alpha^{-1} y^{\prime}\left(\frac{1}{2}-0\right)
\end{array}\right.
$$

where $q(x) \in L_{2}(0,1)$ and $a(\lambda), b(\lambda), c(\lambda)$ and $d(\lambda)$ are unknown coefficients of the problem $L$. Let $\Omega=\left\{x_{n}^{j}\right\} \subset X_{0}$ be the dense subset of nodal points in $(0,1)$ satisfies the following asimptotics

If $x_{n}^{j} \in\left(0, \frac{1}{2}\right)$,

$x_{n}^{j}=\frac{(j+1 / 2)}{n-m-r}+\frac{2+\sin \pi\left(\frac{j+1 / 2}{n-m-r}\right)}{2(n-m-r)^{2} \pi^{2}}+$

$+\frac{2 \alpha^{-}}{\alpha^{+}(n-m-r)^{2} \pi^{2}} \frac{(j+1 / 2)}{n-m-r}+o\left(\frac{1}{n^{2}}\right)$,

If $x_{n}^{j} \in\left(\frac{1}{2}, 1\right)$,

$x_{n}^{j}=\frac{(j+1 / 2)}{n-m-r}+\frac{\sin \pi\left(\frac{j+1 / 2}{n-m-r}\right)}{2(n-m-r)^{2} \pi^{2}}$

$+\frac{2 \alpha^{-}}{\alpha^{+}(n-m-r)^{2} \pi^{2}} \frac{(j+1 / 2)}{n-m-r}+\frac{1-\frac{3 \alpha^{-}}{\alpha^{+}}}{(n-m-r)^{2} \pi^{2}}+o\left(\frac{1}{n^{2}}\right)$.

It can be calculated that

$$
f_{0}(x)=\left\{\begin{array}{c}
1+\frac{1}{2} \sin \pi x+\frac{2 \alpha^{-}}{\alpha^{+}} x, \text { for } x<\frac{1}{2} \\
\frac{1}{2} \sin \pi x+\frac{2 \alpha^{-}}{\alpha^{+}} x+1-\frac{3 \alpha^{-}}{\alpha^{+}} \text {for } x>\frac{1}{2}
\end{array}\right.
$$




$$
\begin{aligned}
& q(x)=2\left[f_{0}^{\prime}(x)-f_{0}(1)+f_{0}(0)+f_{0}\left(\frac{1}{2}+0\right)-f_{0}\left(\frac{1}{2}-0\right)\right] \\
& =\pi \cos \pi x \\
& b_{m}=f_{0}(0)=1, \\
& d_{r}=f_{0}(1)-f_{0}\left(\frac{1}{2}+0\right)+f_{0}\left(\frac{1}{2}-0\right) \\
& -\frac{\alpha^{-}}{\alpha^{+}}\left(b_{m}+\int_{0}^{1 / 2} q(t) \mathrm{d} t\right)=1 .
\end{aligned}
$$

\section{References}

1. P.A. Binding, P.J. Browne and K. Seddighi, Sturm-Liouville problems with eigenparameter dependent boundary conditions, Proc. Edinburgh Math. Soc., 37(2), (1993), 57-72.

2. P.A. Binding, P.J. Browne and B.A. Watson, Inverse spectral problems for Sturm-Liouville equations with eigenparameter dependent boundary conditions, J. London Math. Soc., 62, (2000), 161-182.

3. P.A. Binding, P.J. Browne, B.A. Watson, Sturm-Liouville problems with boundary conditions rationally dependent on the eigenparameter, I, Proc.Edinburgh Math.Soc., 45, (2002), 631-645.

4. P.A. Binding, P.J. Browne, B.A. Watson, Sturm-Liouville problems with boundary conditions rationally dependent on the eigenparameter, II, Journal of Computational and Applied Mathematics, 148, (2002), 147-168.

5. P.J. Browne and B.D. Sleeman, Inverse nodal problem for Sturm-Liouville equation with eigenparameter depend boundary conditions, Inverse Problems 12 (1996), pp. 377-381.

6. Y.H. Cheng, C-K. Law and J. Tsay, Remarks on a new inverse nodal problem, J. Math. Anal. Appl. 248 (2000), pp. 145-155.

7. G. Freiling and V.A. Yurko, Inverse Sturm-Liouville Problems and their Applications, Nova Science, New York, 2001.

8. G. Freiling and V.A. Yurko, Inverse problems for Sturm-Liouville equations with boundary conditions polynomially dependent on the spectral parameter, Inverse Problems, 26, (2010), p. 055003 (17pp.).

9. C.T. Fulton, Two-point boundary value problems with eigenvalue parameter contained in the boundary conditions, Proc. R. Soc. Edinburgh, A77, (1977), 293-308.

10. I.M. Gelfand and B.M. Levitan, On the determination of a differential equation from its spectral function, Amer. Math Soc. Trans. 1 (1951), pp. 253-304.

11. N.J. Guliyev, Inverse eigenvalue problems for Sturm-Liouville equations with spectral parameter linearly contained in one of the boundary condition, Inverse Problems, 21, (2005), 1315-1330.

12. O.H. Hald, Discontinuous inverse eigenvalue problems, Comm. Pure Appl. Math., 37, (1984), 539-577.

13. O.H. Hald and J.R. McLaughlin, Solutions of inverse nodal problems, Inv. Prob. 5 (1989), pp. 307-347.

14. H. Hochstadt and B. Lieberman, An Inverse Sturm-Liouville Problem with Mixed Given Data, SIAM J. Appl. Math. 34 (1978), 676-680.

15. J.R. McLaughlin, Inverse spectral theory using nodal points as data - a uniqueness result, J. Diff. Eq. 73 (1988), pp. 354-362.

16. R. Mennicken, H. Schmid and A.A. Shkalikov, On the eigenvalue accumulation of Sturm-Liouville problems depending nonlinearly on the spectral parameter, Math. Nachr., 189, (1998), 157-170.

17. A.S. Ozkan, B. Keskin, Inverse nodal problems for Sturm-Liouville equation with eigenparameter-dependent boundary and jump conditions, Inverse Problems in Science and Engineering, 23(8), (2015), 1306-1312.

18. Chung-Tsun Shieh and V. A. Yurko, Inverse nodal and inverse spectral problems for discontinuous boundary value problems, J. Math. Anal. Appl. 347 (2008) 266-272.

19. A.A. Shkalikov, Boundary value problems for ordinary differential equations with a parameter in the boundary conditions. J. Sov. Math., 33, (1986), 1311-1342, Translation from Tr. Semin. Im. I.G. Petrovskogo, 9, (1983), 190-229.

20. Xeu-Feng Yang, A solution of the nodal problem, Inverse Problems, 13, (1997) 203-213.

21. Chuan-Fu Yang and Xiao-Ping Yang Inverse nodal problems for the Sturm-Liouville equation with polynomially dependent on the eigenparameter, Inverse Problems in Science and Engineering, 19(7), (2011), 951-961.

22. Chuan-Fu Yang, Inverse nodal problems of discontinuous Sturm-Liouville operator, J. Differential Equations, 254, (2013) 1992-2014.

23. V.A. Yurko, Boundary value problems with a parameter in the boundary conditions, Izv. Akad. Nauk Armyan. SSR, Ser. Mat., 19(5), (1984), 398-409, English translation in Soviet J. Contemporary Math. Anal., 19(5), (1984), 62-73. 